\title{
Influence of the Centaurs and TNOs on the main belt and its families
}

\author{
Mattia A. Galiazzo ${ }^{1,2,3} \cdot$ Paul Wiegert ${ }^{1,2} \cdot$ Safwan Aljbaae $^{4}$
}

Received: 1 July 2016 / Accepted: 11 October 2016 / Published online: 8 November 2016

(C) Springer Science+Business Media Dordrecht 2016

\begin{abstract}
Centaurs are objects whose orbits are found between those of the giant planets. They are supposed to originate mainly from the Trans-Neptunian objects, and they are among the sources of Near-Earth Objects. Trans-Neptunian Objects (TNOs) cross Neptune's orbit and produce the Centaurs. We investigate their interactions with main belt asteroids to determine if chaotic scattering caused by close encounters and impacts by these bodies may have played a role in the dynamical evolution of the main belt. We find that Centaurs and TNOs that reach the inner Solar System can modify the orbits of main belt asteroids, though only if their mass is of the order of $10^{-9} m_{\odot}$ for single encounters or, one order less in case of multiple close encounters. Centaurs and TNOs are unlikely to have significantly dispersed young asteroid families in the main belt, but they could have perturbed some old asteroid families. Current main belt asteroids that originated as Centaurs or Trans-Neptunian Objects may lie in the outer belt with short lifetime $\leq 4 \mathrm{My}$, most likely between 2.8 and 3.2 au at larger eccentricities than typical of main belt asteroids.
\end{abstract}

Keywords Minor planets - Asteroids: general - celestial mechanics

M.A. Galiazzo

mgaliazz@uwo.ca; mattia.galiazzo@univie.ac.at

1 Department of Physics and Astronomy, The University of Western Ontario, London, ON, N6A 3K7, Canada

2 Institute of Astrophysics, University of Vienna, Turkenschanzstr. 17, 1180 Vienna, Austria

3 Centre for Planetary Science and Exploration (CPSX), London, ON, N6A 3K7, Canada

4 UNESP, Grupo de dinâmica Orbital e Planetologia, Univ. Estadual Paulista, Guaratinguetá, SP, 12516-410, Brazil

\section{Introduction}

Centaurs are objects whose orbits are contained between those of Jupiter and Neptune (Gladman et al. 2008). Currently, ${ }^{1} 301$ Centaurs are known (JPL Small-Body Database Search Engine, JPL-SBDSE, at http://ssd.jpl.nasa.gov/ sbdb_query.cgi) and recent observational results estimate a value of $240 \pm 130$ with an absolute magnitude $H<$ 10 (Adams et al. 2014). The Centaurs' population with a diameter larger than $1 \mathrm{~km}$ is estimated to be between $\sim 10$ million (Volk and Malhotra 2013) and about $\sim 8 \times$ $10^{9}$ (Fernández et al. 2004; di Sisto and Brunini 2007; Napier 2015). No well-characterized survey has been carried out to date that would allow derivation of a de-biased Centaur size distribution (Fuentes et al. 2014). They are thought to originate mainly from the Trans-Neptunian objects (Levison and Duncan 1993, 1997; Tiscareno and Malhotra 2003; Lykawka et al. 2009; di Sisto et al. 2010; Brasser et al. 2012) and they are among the sources of NearEarth Objects (Morbidelli 1997; Levison and Duncan 1997; Tiscareno and Malhotra 2003; di Sisto and Brunini 2007). Since some Centaurs and their progenitors can be relatively massive $^{2}\left(m \gtrsim 10^{-9} m_{\odot}\right.$, of the order of one-tenth of the lunar mass, $\sim 4 \times 10^{-8} m_{\odot}$ ), move throughout the planetary system, and have done so throughout their existence (also in much larger numbers in the past), we ponder whether close encounters and possible impacts by them on main belt asteroids may have played a role in the recent (that is, after the Late Heavy Bombardment, from 3.8 Gyrs ago to now) dy-

\footnotetext{
${ }^{1}$ June 2015.

${ }^{2}$ The majority of the observed Centaurs and TNOs have smaller masses, e.g. for Schwamb et al. (2014), large bodies comparable to Pluto in size (and so masses) are $\sim 12$, but in the past there were many more of them (Brasser and Morbidelli 2013).
} 
namical evolution of the main belt and particularly asteroid families.

In order to do this, we consider two time spans. The first, which we call present population (PP), reaching $50 \mathrm{Myr}$ into the past, examines the effect of Centaur encounters on young asteroid families, i.e. the Karin family (5.3 Myr, Nesvorný et al. 2002). The second, which we will call the ancient population, AP, stretches back 3.8 Gyrs ago (Hartmann et al. 2000; Kirchoff et al. 2013), the estimated age of the end of the Late Heavy Bombardment (LHB) process, and examines the effects on old asteroid families, e.g. Flora family, which is old $\sim 4.4$ Gyr (Carruba et al. 2016). In particular, we plan to investigate if close encounters with Centaurs and TNOs (from now on C+TNOs), with a diameter larger than $100 \mathrm{~km},{ }^{3}$ could have been responsible for perturbing or diffusing young and/or old main belt families (Zappalà et al. 1995; Migliorini et al. 1995; Nesvorny 2012; Novaković et al. 2011), an example is the scattering of Vtype (basaltic) asteroids from the Vesta family beyond the $3 \mathrm{~J}: 1$ mean motion resonance, into the central and outer main belt, see also Carruba et al. (2014) and Huaman et al. (2014). We also consider whether Centaurs contribute to the presence of interlopers inside families, like the case of the C-types (carbonaceous asteroids) in the Hungaria family (up to $6 \%$, Warner et al. 2009), whose member are in majority E-types.

Centaur orbits are dynamically unstable, with a dynamical lifetime from less than 1 to about $100 \mathrm{Myr}$ (Horner et al. 2004a; Tiscareno and Malhotra 2003; Bailey and Malhotra 2009). In fact, their dynamical evolution is mostly influenced by close encounters with giant planets (as found in Horner et al. 2004a, 2004b).

Their lifetimes are short in general compared to the lifetime of main belt asteroids because Centaurs are mostly in very chaotic regions perturbed by the giant planets. According to Bailey and Malhotra (2009), the lifetime of the Centaurs (at least the most chaotic ones, the majority) is about 22 Myrs; Dones et al. (1996) assert $~ 5 \mathrm{Myr}$; and up to $72 \mathrm{Myr}$ for di Sisto and Brunini (2007). Hence, they are typically removed from the Solar System on timescales of only millions of years (Levison and Duncan 1997; Tiscareno and Malhotra 2003; Horner et al. 2004a and previously cited papers). Their lifetime typically ends by ejection on a hyperbolic orbit or collision with a planet (Horner et al. 2004a, 2004b; di Sisto et al. 2010; Galiazzo 2013). Some may become short-period comets and a fraction of them may impact the terrestrial planets or the Sun.

The Centaur progenitors, the TNOs, are mainly subdivided into 4 principal regions (Adams et al. 2014): (i) Res-

\footnotetext{
${ }^{3}$ The diameter was computed using the equation of Tedesco et al. (1992), assuming the average albedo $\rho_{V}=0.05$ of the Centaurs (Rabinowitz et al. 2007): $D=\frac{1329}{\rho_{V}} 10^{-\frac{H}{5}}$. In this way, we can assume a body is larger than $100 \mathrm{~km}$ when its absolute magnitude, $H \lesssim 9$.
}

onant objects, bodies which occupy a mean-motion resonance with Neptune, e.g. the Plutinos (objects in N2:3 resonance with Neptune); (ii) Scattered Near objects, which have a semi-major axis that vary of $\delta a=\frac{a_{\max }-a_{\min }}{\langle a\rangle} \geq 0.02$, over 10 Myr time scales; (iii) Scattered Extended objects, bodies with $s=\sqrt{e^{2}+\sin ^{2}(i)} \geq 0.25$, where $e$ and $i$ are respectively the eccentricity and the inclination; (iv) Classical Objects, which have $s<0.25$. All the dynamically excited objects with a semi-major axis, $a_{\mathrm{Nep}} \lesssim a \lesssim 80$ au are grouped together in one single Scattered class. In this work we consider only the TNOs with a semi-major axis, $a \lesssim 80$ au, a relatively well-characterized source region (Adams et al. 2014) inside the heliopause.

This paper is organized as follow: the model is described in Sect. 2. Section 3 is subdivided into 2 parts: the semianalytical and analytical studies in Sect. 3.1, and the numerical results which we revisit in Sect.3.2. Finally, our conclusions are given in Sect. 4.

\section{Model}

To investigate how these large bodies might perturb asteroids in the main belt (MBAs), we performed a numerical simulation of the orbital evolution of the C+TNOs through the main belt using the Lie-integrator (Hanslmeier and Dvorak 1984). This orbital numerical integrator has an adaptive step-size (Eggl and Dvorak 2010; Bancelin et al. 2012) and it was used in several previous works dealing with close encounters of asteroids with the planets (Galiazzo 2013; Galiazzo et al. 2013a, 2013b, 2014; Galiazzo and Schwarz 2014). In this work we use an accuracy parameter set to $10^{-13}$. The output stepsize for the numerical integration is set to $1 \mathrm{kyr}$ and a simplified solar system (SSS: all planets from Venus to Neptune, with the mass of Mercury added to the mass of the Sun) is considered for the orbital propagation. We assume a close encounter (CE) is occurring when a body is within $\Delta r_{\mathrm{CE}}=0.0025$ au from the perturbing body. Elements of the two bodies are registered, and the perturbation is computed from the moment the asteroid enters into this sphere of radius $\Delta r_{\mathrm{CE}}$ around the massive body until the moment that it exits. We chose a $\Delta r_{\mathrm{CE}}$ value roughly in the proximity of the Hill's sphere radius, considering the largest Centaur: Centaurs Hill spheres can reach $R_{H} \sim a(1-e)\left(m_{c} / m_{\odot}\right)^{1 / 3} \sim 2 \times 10^{-3}$ au, which is the Hill sphere for the largest Centaur $1995 \mathrm{SN}_{55}$.

The orbital evolution of a body is considered until it either escapes or it collides. We consider an escape when the body has an instantaneous eccentricity $e>0.99$, its period exceeds $1000 \mathrm{yrs}$ and $a \gtrsim 80 \mathrm{au}$. Then in order to consider the influence of a massive $\mathrm{C}+\mathrm{TNO}$ in the belt, we check its influence if its perihelion drops below 3.8 au and if it remains there for at least 2 kyrs ( 2 output times steps of our 
simulations). For each $\mathrm{C}+\mathrm{TNO}$ that meets this criterion in our initial simulations, we make a second integration with a finer output interval for the entire period that the body was crossing the asteroid belt, using the elements of the $100-\mathrm{km}$ sized $\mathrm{C}+\mathrm{TNO}$ just as it enters the main belt from the first integration.

This finer integration proceeds for the duration of the C+TNO's residence in the main belt, as long as its perihelion is in the range $1.78<q<3.8$ au. This outer border is chosen near the aphelion of $522 \mathrm{Helga},{ }^{4}$ the main body of the outermost asteroid family (Carruba et al. 2015) and the inner one is the beginning of the Hungaria family (Galiazzo et al. 2013a), the closest main belt family to Mars. During the finer integration, all close approaches to asteroids are examined in order to determine the perturbation to the asteroid's orbit.

A main belt family can potentially lose one of its members once a close encounter causes the asteroid (member of the family) a real semi-major axis change of more than 0.0003 au (from now on $K_{\text {kill }}$ ), because this is enough to affect the proper semi-major axis at a level that may affect the apparent membership of the asteroid in an asteroid family (Knežević and Milani 2003). We will assume that the main belt (and so the asteroid belt families) is significantly perturbed by the C+TNOs if at least $\sim 3-5 \%$ of the members of the main belt are deflected by an amount $\Delta a \geq K_{\text {kill }}$.

The effects of the $\mathrm{C}+\mathrm{TNO}$ on the asteroid belt are determined from their perturbations on a sample asteroid population. We want to check in particular if the TNOs are capable of scattering members of the Vesta family, and therefore explain the presence of some V-types in other families of the belt. So we divide the asteroid belt into 2 sub-samples:

1. A complete known Vesta family (Vestoid elements were taken from the DPS catalogue, Nesvorny 2012: 528 Vestoids);

2. A sample of the remaining (non-Vestoid) main belt asteroids: only a sub-sample of the remaining asteroids was propagated in order to save computational time. This sample has 1054 bodies, proportionally distributed among the respective different regions. For our purposes, the main belt is divided into 6 regions, based on semimajor axis (Table 1) and inclination $\left(i=17.16^{\circ}\right.$, this value distinguishes the high inclined asteroid families from the low ones, as suggested in Novaković et al. 2011) similarly to Galiazzo et al. (2013b).

For the size distribution of the main belt asteroids, we considered only bodies with diameter as large or larger than $1 \mathrm{~km}$ (with $H<14$, asteroids with $D \approx 4-9 \mathrm{~km}$,

\footnotetext{
${ }^{4}$ In practice, we examined all those below a value of 3.91 au (Helga's aphelion), to be sure that the Centaurs and TNOs interact with this region.
}

Table 1 Subdivision of the Main belt in different regions in $(a-i)$ space (semi-major axis interval $(\Delta a)$ and inclination $(i)$ ). The number of asteroids (considered in the orbital integrations) which belong to each region is given: "\# low" (low inclinations) stands for the number of asteroid in the relative semi-major axis subregion with inclinations $i \leq 17.16^{\circ}$ and "\# high" (high inclinations) vice-versa, $i>17.16^{\circ}$. The most populated region is the central belt. MB stands for main belt, $I$, $M$ and $O$ stand respectively for Inner, Middle and Outer

\begin{tabular}{llll}
\hline GROUP & $\Delta a[\mathrm{au}]$ & \# low & \# high \\
\hline IMB & $1.78 \leq a \leq 2.5$ & 146 & 29 \\
MMB & $2.5<a \leq 2.83$ & 306 & 19 \\
OMB & $2.83<a \leq 3.8$ & 476 & 72 \\
\hline
\end{tabular}

see http://www.minorplanetcenter.net/iau/Sizes.html) in order to have a representative sample quantitatively large enough to make a good statistical study, but that would not cost too much in terms of computational effort. Asteroids in this size range are large enough that we can propagate their orbits in the belt with only gravitational forces for the small amount of time (usually $t<0.5 \mathrm{Myrs}$ ) a C+TNO typically interacts with the main belt. Osculating elements are taken from the JPL-SBDSE which give in total 26711 MBAs asteroids with $H<14$ and, as we said before, from them we take a reasonable proportional distributed sub-sample for the non-Vestoids and all the Vestoids in this size-range.

\subsection{Present and past population}

As mentioned earlier, we consider 2 populations in order to study the effect on the present solar system and on the past population just after the LHB (post-LHB).

For the $P P$ we take as a sample the observed $\mathrm{C}+\mathrm{TNO}$ larger than $100 \mathrm{~km}$ in diameter ${ }^{5}$ (shown in the $a-e$ and $a-i$ spaces in Figs. 1 and 2), for which we will assume the observational bias should be negligible. For the $A P$, we create a debiased population based on the work of Adams et al. (2014) and assume a number of bodies appropriate to 3.8 Gyrs ago using the work of Brasser and Morbidelli (2013). In both cases we take the SSS model.

PP We study the orbital evolution of the present known Centaurs and TNOs for 50 Myrs. We consider the known objects whose orbits are in an interval of semi-major axis $5.5<a \leq 80 \mathrm{au}$, and perihelion $q \leq 40 \mathrm{au}$. Tiscareno and Malhotra (2003) consider $q=33$ au a good boundary for TNOs having a similar dynamical lifetime to the Centaurs, but in order to be sure, we go further in semi-major axis range till $40 \mathrm{au}$. The $P P$ sample is shown in $a-e$ and $a-i$

${ }^{5}$ Computed via the Tedesco et al. (1992) equation, assuming the average albedo $\rho_{V}=0.05$ of the Centaurs (Rabinowitz et al. 2007): $D=\frac{1329}{\rho_{V}} 10^{-\frac{H}{5}} . H$ is the absolute magnitude, and $D \gtrsim 100$ when $H \lesssim 9$. 

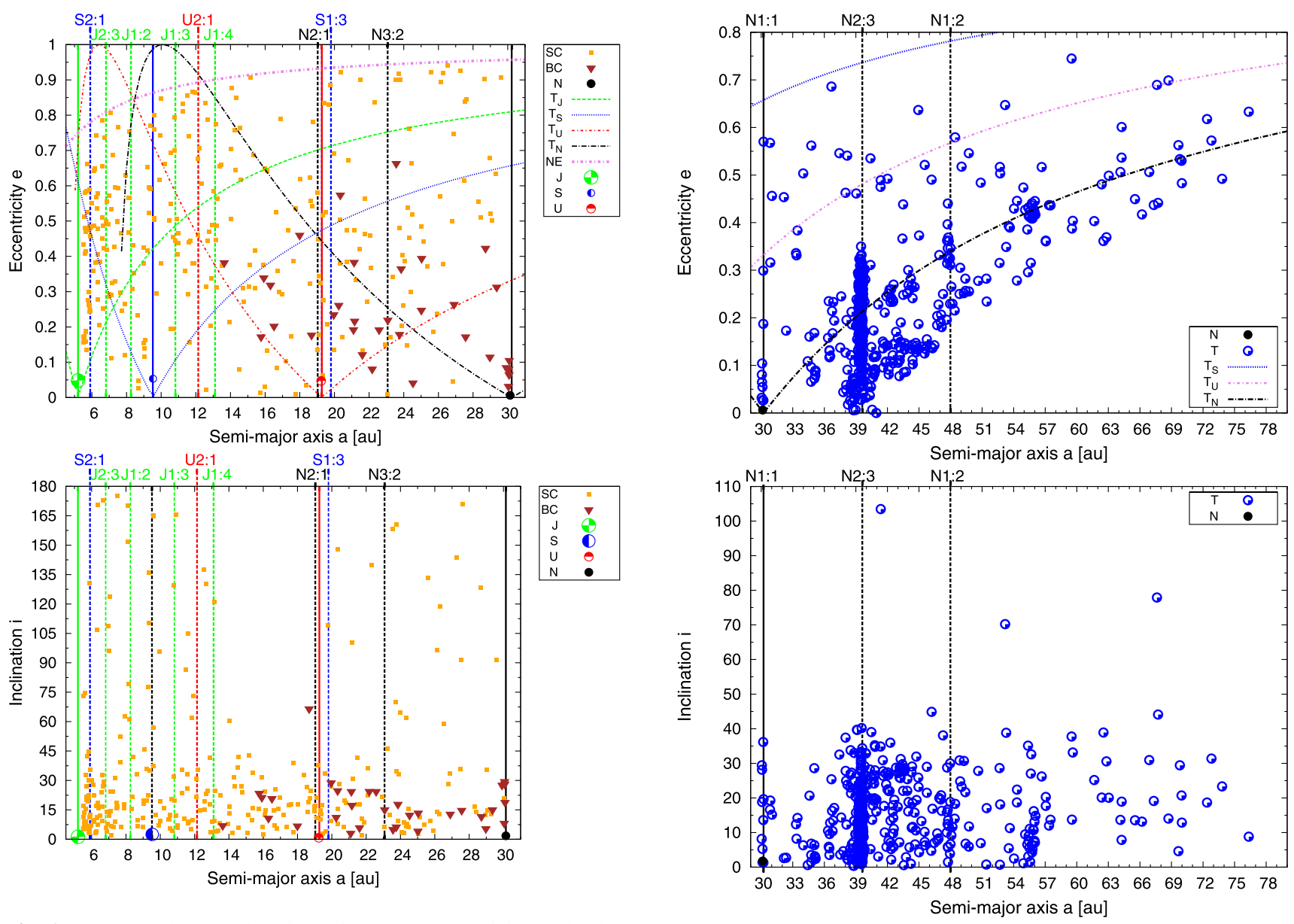

Fig. 1 (Top panel) A semi-major axis versus eccentricity projection of "small" Centaurs (SC) and $D>100 \mathrm{~km}$ Centaurs (BC). Vertical lines are the principal mean motion resonances (continuous lines are only for $1: 1$ ) for Centaurs with respect to the giant planet, represented by the filled circles whose symbols have sizes proportional to the real diameter of the planets (Jupiter (J), Saturn (S), Uranus (U) and Neptune $(\mathrm{N})$ ). The top curve (NE) is the border between the NEAs and the Centaurs. Other curved lines are the regions strongly dominated by the gravitational influence of the giant planets, represented by the Tisserand parameter curves $\left(T_{\mathrm{J}}, T_{\mathrm{S}}, T_{\mathrm{U}}\right.$ and $T_{\mathrm{N}}$, respectively for Jupiter, Saturn, Uranus and Neptune). (Bottom panel) A semi-major axis versus inclination projection of Centaurs

spaces in Fig. 1. The orbital elements of these bodies were taken from the JPL-SBDSE. We then create 10 clones for each Centaur, and 5 for each TNO with these osculating elements: $a=a_{0} \pm 0.005, e=e_{0} \pm 0.003$ and $i=i_{0} \pm 0.01$, similarly to Horner et al. (2004a), where $a_{0}, e_{0}$ and $i_{0}$ are the initial elements. These clones are evolved over 50 Myrs in the SSS during which we check which bodies reach the main belt region, and how many asteroids and which main belt families are perturbed.

AP Schwamb et al. (2014) estimates that the total number of the large bodies $\left(m>10^{-9} m_{\odot}\right.$, which we take to mean those bodies with visual $r$-magnitude less than 19.5, e.g. Gladman et al. 2001), we are interested in is 12 bodies

Fig. 2 (Top panel) A semi-major axis versus eccentricity projection of TNOs with $D>100 \mathrm{~km}(\mathrm{~T})$. Vertical lines represent the principal mean motion resonances with Neptune $(\mathrm{N}) . T_{\mathrm{S}}, T_{\mathrm{U}}$ and $T_{\mathrm{N}}$ are like in Fig. 1. (Bottom panel) A semi-major axis versus inclination projection of TNOs $(D>100 \mathrm{~km})$ orbits

of which 9 are currently known. Extrapolating from the results from Brasser and Morbidelli (2013), we anticipate that 3.8 Gyrs ago, the TNO population was about 17 times more numerous than now. So we take the size of the ancient population to be $12 \times 17=204$ TNOs here. Eris' mass is picked as representative of the largest sizes expected in the primordial Kuiper Belt, $m_{\text {Eris }}=1.66 \times 10^{22} \mathrm{~kg} \sim 8 \times 10^{-9} m_{\odot}$ (Brown and Schaller 2007).

We simulate this ancient population assuming the debiased orbital distribution of Adams et al. (2014). This assumes that the current distributions in sub-groups of the TNOs are in the same proportions as the post-LHB ones. From the Deep Ecliptic Survey (DES), Adams et al. (2014) computed that only 3 classes of TNOs can contain objects as massive as we desire: the Classical, the Scattered and the $3: 2$ resonant. These relative populations of large objects (at least at $H<4$ ) can be approximated by a $2: 2: 1$ ratio among them, which we assume represents approximately the post-LHB situation. We choose a number of $\mathrm{C}+\mathrm{TNO}$ 
clones at the previous ratios in sufficient numbers to have a reasonable statistical sample among the principal osculating elements ( $a, e$ and $i$ ), following Adams et al. (2014). Our initial population was 170 clones for the Classical and Scattered population and 85 for the $3: 2$ population.

We integrate the orbits of this synthetic population for 200 Myrs.

\section{Interactions with the main belt asteroids: numerical estimates of the diffusion into the inner solar system}

We now describe the numerical results considering the present and the ancient populations:

PP C+TNOs start to cross Jupiter's orbit and enter in the main belt region after experiencing close encounters (exciting their eccentricity) with the giant planets: Jupiter is the most important for the Centaurs, and Neptune for the TNOs. It is interesting to note that initially the largest Centaurs' orbits are beyond Saturn and in particular beyond the J4 : 1 resonance (Fig. 1).

We find in the numerical integrations that solely those with $q<34$ au can enter in the main belt region in 50 Myrs of evolution, with the exception of $\mathrm{C}+\mathrm{TNO}$ in resonance like $\mathrm{N} 2: 3$, which can have larger perihelia.

In Fig. 3, we can see 2 examples of 100-km size bodies that enter the belt, passing through it for some kyrs and eventually escaping: a Centaur (the first one which escapes) and a TNO (a Kuiper Belt Object). The third (and last) object, in Fig. 3, which crosses Jupiter's orbit is initially a member of the Scattered Disk which arrives only next to Jupiter's orbit after an important close encounter with Saturn and is ejected out of the Solar System.

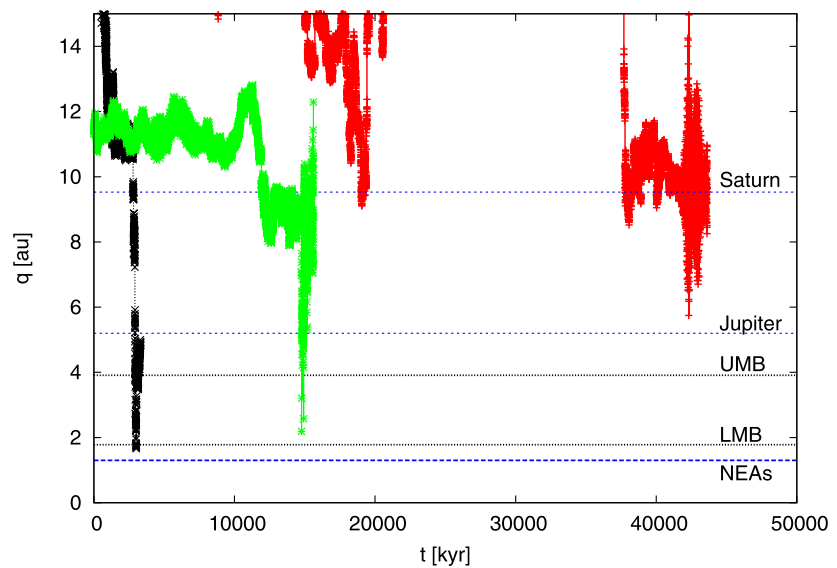

Fig. 3 Perihelia of a sample of $100 \mathrm{~km}$-size bodies which cross Jupiter' orbit, during their 50 Myr evolution and eventually enter in the main belt. $\mathrm{UMB}=$ Upper border of the Main Belt, $\mathrm{LMB}=$ Lower border of the Main Belt, NEAs = Near-Earth Objects
The majority of the Centaurs that enter into the Main Belt pass through in the first $\sim 10 \mathrm{Myr}$ (average value) and stay there for about $\sim 120 \mathrm{kyr}$.

They usually end as Sun-grazers or collide with the Sun or escape. Some stay in the belt for as long as $\sim 3 \mathrm{Myr}$.

TNOs reach the asteroid belt later (as expected, since they are Centaur progenitors), at $\sim 17 \mathrm{Myr}$ (average time) and reside there for a period of time similar to the Centaurs' one. In general, C+TNOs can arrive through all the 50 Myrs of integration. 240 Centaur clones out of 1023 enter the main belt, and 73 TNOs out of 2871, respectively $23 \%$ and $\sim 3 \%$. Table 2 shows the time of residence in the main belt.

The $\mathrm{C}+\mathrm{TNO}$ s that stay there the least time usually experience several close encounters with Jupiter, which modify their orbits substantially (see previous section). The Centaurs which enter in the main belt are those ones that start their journey outside Saturn: their initial semi-major axis is $a \gtrsim 13.7 \mathrm{au}$. Some Centaurs are found to have a very high probability to interact with the belt, i.e. $2003 \mathrm{QC}_{112}$ and also the largest one, $1995 \mathrm{SN}_{55}$.

About 100 MBAs out of 1582 have close encounters with TNOs $(\sim 6 \%)$ and only $5(<1 \%)$ of these produce a drift of the order of $10^{-4} \mathrm{au}$. There are also a few cases where asteroids have multiple deflections of the order of $10^{-5}$ au, by the same $\mathrm{C}+\mathrm{TNO}$ (even though their mass is only of the order of $\left.10^{-10} m_{\odot}\right)$.

AP From our numerical integrations, we find that in 200 Myrs only objects from the Classical and 3:2 populations can enter in the main belt. They do so after entering in the Centaur region and interacting with the giant planets.

The synthetic TNOs in this sample which reach the main belt stay about $1 / 4$ million years. $8.5 \%$ (36 out of 225 ) of the TNOs simulated enter the main belt in $200 \mathrm{Myrs}$, and a good number of them $(\sim 50 \%$ of the ones which enter $)$ provide an effective perturbation on the MBAs, see the histograms with the lowest rates in Fig. 4. This last figure shows that the main belt entry rate of TNOs (the $\mathrm{TNOs}_{M}$ ) and the sub-group of them which not only enters but also makes an effective perturbation on MBAs ( $\mathrm{TNOs}_{M^{\prime}}$ ) is similar, meaning that the large bodies which make a scattering and the ones which do not, have a similar decay in population.

Table $2 \mathrm{C}+\mathrm{TNO}$ stal life (Myrs) in the main belt (maximum, $T_{\max }$ and average value, $\langle T\rangle)$ and (average) arrival time $\left(T_{\mathrm{a}}\right)$. The symbol $*$ underlines that the synthetic populations of TNOs $(A P)$ was integrated for longer time than the present population $(P P), 200$ Myrs instead of 50 Myrs

\begin{tabular}{llll}
\hline Group & $T_{\max }$ & $\langle T\rangle$ & $T_{\mathrm{a}}$ \\
\hline Centaurs $(P P)$ & 3.1 & 0.1 & 10.0 \\
TNOs $(P P)$ & 1.9 & 0.1 & 16.8 \\
TNOs $(A P)^{*}$ & 3.7 & 0.2 & 85.2 \\
\hline
\end{tabular}




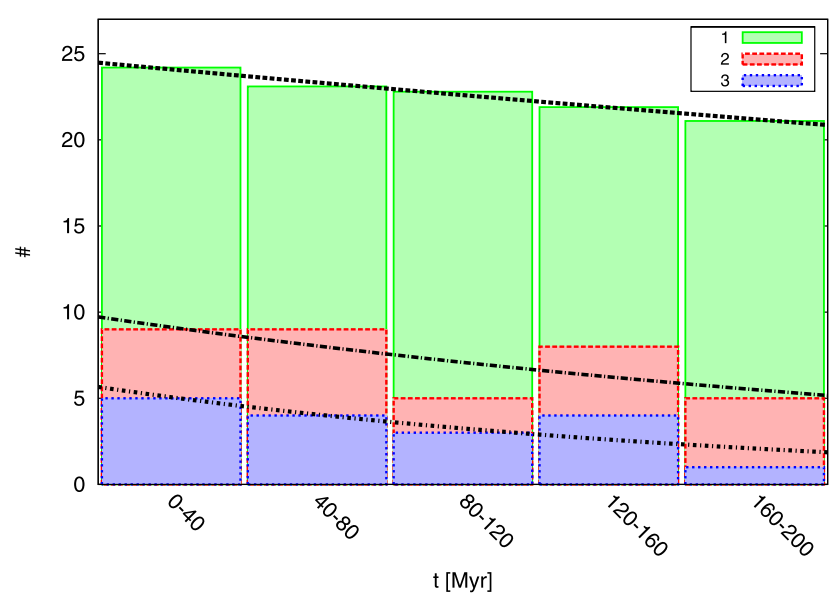

Fig. 4 Number of TNOs (\#) per interval of time $(t)$ : rate every 40 Myrs. The histogram represents 3 populations rates: (i) surviving TNOs (columns with the highest rates. This rate was scaled by 10 in order to show better the trend of the other 2 groups) for the classes which contribute to the scattering in the main belt: Classical and N2:3 classes; (ii) TNOs entering the main belt; and (iii) TNOs entering the main belt which provide a $\Delta a>K_{\text {kill }}$. Each group is fitted to its own decay function

Also shown is the rate at which TNOs (the 'survivors', TNOs $_{S}$ : all the TNOs which did not have ejections or impacts). Fitting the trends, with an exponential decay function, $N_{\mathrm{TNO}}=N_{0} e^{-t / \lambda}$, we can compute their half-life $\left(t_{1 / 2}=\lambda \ln 2\right): t_{1 / 2, \mathrm{TNO}_{M}}=215 \pm 132 \mathrm{kyr}$ and $t_{1 / 2, \mathrm{TNOs}_{M^{\prime}}}=$ $125 \pm 28$ kyrs. The decay rate of TNOs $_{S}$ is at least 3 times slower, $t_{1 / 2, \mathrm{TNO}_{S}}=847 \pm 69 \mathrm{kyrs}$, however, all rates are of the same order.

Concerning the most perturbed main belt asteroids, that is, those with $\Delta a \gtrsim K_{\text {kill }}$, we find 66 deflections this large out of the 261 recorded, $\sim 25 \%$. The largest reaches $\sim 10$ times $K_{\text {kill }}$. Of these deflections, 7 were Vestoids (V, $\left.N_{\mathrm{V}}=7\right)$ and 59 non-Vestoids $\left(\mathrm{NV}, N_{\mathrm{NV}}=59\right)$. In order to compute the real number of asteroids with $H<14$ deflected in 200 Myrs, we have to scale these numbers by the real number of TNOs 3.8 Gyrs ago. In the case of the $\mathrm{NV}$, also by the real total number of the main belt NV with $H<14, N_{\mathrm{NV}, \mathrm{T}}=26183$ : (the total number of non-Vestoids with $H<14$, see Sect. 2).

The total number of deflected asteroids will be the sum of the total $\mathrm{V}\left(N_{\mathrm{T}, \mathrm{V}, \text { def }}\right)$ and $\mathrm{NV}\left(N_{\mathrm{T}, \mathrm{NV} \text {,def }}\right)$ deflected:

$$
\begin{aligned}
N_{\mathrm{T}, \mathrm{def}} & =N_{\mathrm{T}, \mathrm{V}, \mathrm{def}}+N_{\mathrm{T}, \mathrm{NV}, \mathrm{def}} \\
& =N_{\mathrm{V}} S_{1}+N_{\mathrm{NV}} S_{2} S_{1} \\
& =\frac{N_{\mathrm{V}} N_{\mathrm{T}, 1}}{N_{\mathrm{deb}}}+N_{\mathrm{NV}} \frac{N_{\mathrm{NV}, \mathrm{T}}}{N_{\mathrm{NV}, \mathrm{S}}} \frac{N_{\mathrm{T}, 1}}{N_{\mathrm{deb}}}=707
\end{aligned}
$$

where $N_{\mathrm{NV}, \mathrm{S}}$ is the total number of $\mathrm{NV}$ in the sample used for the numerical integrations. Then $N_{\mathrm{T}, 1}=204(12 \times 17)$ is the total number of the most massive $\left(m>10^{-9} m_{\odot}\right)$ TNOs at 3.8 Gyrs ago and $N_{\text {deb }}$ is the number of synthetic TNOs considered for the orbital evolution of the debiased population $\left(N_{\mathrm{deb}}=170+170+85=425\right) . S_{1}$ and $S_{2}$ (where $1=$ TNOs and $2=$ MBAs) are the appropriate scaling factors for quantities respectively dependent on the real number of TNOs and of MBAs. Having $707 \mathrm{~km}$-size asteroids deflected out of their families means that about $3 \%$ of the MBAs have their orbits significantly changed by the passage of the C+TNOs in 200 Myrs, and in particular the percentage, therefore the perturbation, will be higher in specific regions of the main belt (so also in some specific family), see Sect. 3.2 and in particular Fig. 6.

\subsection{Typical dynamical orbit of the belt-crossing Centaurs and TNOS}

The $\mathrm{C}+\mathrm{TNO}$ initially interact more with the outer main belt than the inner regions of the belt. Figure 5 shows this behavior clearly. They have semi-major axes which spread from the main belt to outer regions larger than 5 au. Only a small number of them has $i>17.16^{\circ}$; however, as shown in Fig. 6, some families at high inclinations can be influenced by the TNOs. The $\mathrm{C}+\mathrm{TNOs}$ arrive in the main belt at high eccentricities (in comparison with typical MBAs), generally $e \gtrsim 0.16$, and inclinations, $i \lesssim 25^{\circ}$, with only one case at $i=36^{\circ}$. In particular the typical semi-major axis of the Ancient Population, when arriving at the belt, favors the outer belt beyond 3.4 au, while on the contrary the Present Population can arrive also in the middle main belt. For these large bodies entering in the belt, the average and median values of their semi-major axes are respectively $(5.7 \pm 2.6)$ and 5.0 au in the $P P$ of Centaurs, and $(5.7 \pm 4.8)$ and 4.9 au of TNOs, respectively. However, considering the whole Centaurs and TNOs, these values become $(5.7 \pm 3.6)$ and 4.9 au in the $P P$ and $(4.9 \pm 1.2)$ and 4.4 au in the $A P$.

The ancient population is the most important one in terms of scattering MBAs out of their relative main belt families. The most perturbed regions of the belt are shown in the lower panel of Fig. 5, which illustrates the perturbed region of the $A P$ by a line connecting aphelion and perihelion. The most influenced region is the Outer main belt at low inclinations.

The next section will show in more detail which asteroid families are most affected. Here, in particular we want to underline that the $A P$ can reach inclinations up to $23^{\circ}$ and have a perihelion in the inner main belt. The AP orbits are usually controlled by Jupiter which also affects their eccentricities through close encounters, and which eventually ejects the $\mathrm{C}+\mathrm{TNOS}$ in hyperbolic orbits or causes them to collide with the Sun.

Although the behavior between the AP and PP is quite similar, the main difference is that on average the Present Population has lower perihelia when it starts to enter in the belt and, in fact, the median eccentricity of the $P P, \bar{e}_{P P}=$ 

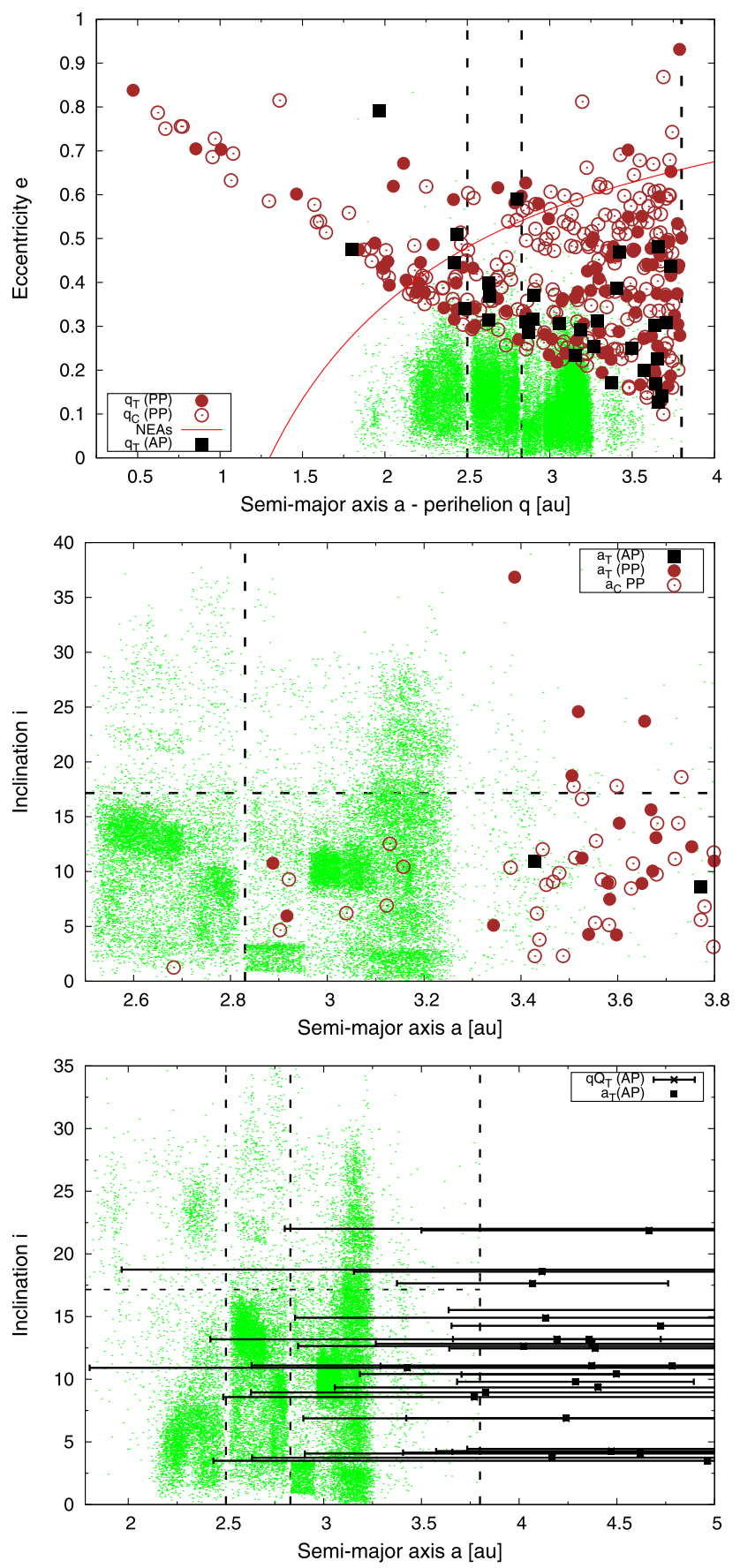

Fig. 5 In each panel, the semi-major axis of the main belt asteroids with $H<14$ is represented with small green dots. The larger points are related to the $\mathrm{C}+\mathrm{TNO}$ populations when they first enter the mainbelt. (Top panel) Overplotted is the perihelion versus eccentricity projection of the Present Population (Centaurs, $q_{\mathrm{C}}(\mathrm{PP})$, and TNOs, $q_{\mathrm{T}}$ $(\mathrm{PP}))$ and of the Ancient Population $\left(q_{\mathrm{T}}(\mathrm{AP})\right)$. Vertical dotted lines are the borders of the main belt regions. The region to the left of the red line is the NEA region. (Middle panel) A semi-major axis versus inclination projection of the Ancient and Present populations. (Bottom panel) Semi-major axis ( $a_{\mathrm{T}}$ with aphelion and perihelion limits, $q Q_{\mathrm{T}}$ ) versus inclination projection of the TNOs of the Ancient Population
0.39 , is higher than the one of the $A P, \bar{e}_{A P}=0.31$. This also means that the $A P$ has more possibility to encounter Main belt asteroids, because the related orbits intersect those of the MBAs for longer time.

Finally, we note that some ${ }^{6}$ Centaurs and TransNeptunian Objects reach similar orbits to those of the present main belt asteroids (see in particular the middle panel of Fig. 5) and thus there could be Centaurs and/or TNOs interlopers in the main belt (as suggested, e.g. by Fernández and Sosa 2015). Similar orbits to our simulated $\mathrm{C}+\mathrm{TNO}$ interacting with the belt are in particular the orbits such as asteroids (511) Davida, (52) Europa, (31) Euphrosyne, and (24) Themis in the outer belt.

\subsection{Can C+TNOs diffuse asteroid families?}

The effect of asteroidal diffusion by $\mathrm{C}+\mathrm{TNO}$ is very small for the case of young families (the $P P$ results): only one case of a deflection over $K_{\text {kill }}$ by a TNO was recorded.

Therefore, perturbations by the $\mathrm{C}+\mathrm{TNO}$ s have a negligible effect on asteroid families for the current main belt. In the $A P$ case, there is more time ( $3.8 \mathrm{Gyr}$ ) for $\mathrm{C}+\mathrm{TNO}$ to be effective at disrupting asteroid families, and so older families might still be affected. Numerical integrations show that the time spent by individual $\mathrm{C}+\mathrm{TNO}$ s within the main-belt is different from past to present, at least the double of the average for the $A P$ versus the $P P$. This can be seen in the orbital evolution of our TNO population (Fig. 4) and was shown also by Brasser and Morbidelli (2013). Most of the $A P$ bodies enter the main belt sooner with lower eccentricity than $P P$ (see Sect. 3.1), so they can spend more time in the belt and with a smaller encounter velocity, which helps to increase the deflection of the asteroids. The TNO population decay also explains why we do not see important perturbations of the main belt now: the TNOs that pass now through the solar system are on more eccentric orbits than in the past.

Because the fraction of MBAs affected over $3.8 \mathrm{Gyr}$ at the current rate would be at least $3 \%$, this effect can not be neglected in the long-term. $\mathrm{C}+\mathrm{TNO}$ reached the mainbelt in much increased numbers in the past and so they have participated in the disruption and diffusion of very old families. Different main belt families are expected to have experienced the deflection of at least 100 family members with sizes of the order of one $\mathrm{km}$ beyond the cutoff $\Delta a \gtrsim K_{\text {kill }}$ due to the influence of the passage of the ancient population, and these are: Hygiea (the most perturbed one if we consider also asteroids affected at the

${ }^{6}$ For the Present Population, $16 \%$ of the Centaurs and $18 \%$ of the TNOs, for the Ancient Population $6 \%$. The rest of all those populations which interact with the main belt (having perihelion, $q \lesssim 3.8$ for at least 2 kyrs, see Sect. 2) have semi-major axis $a>3.8$ au. 


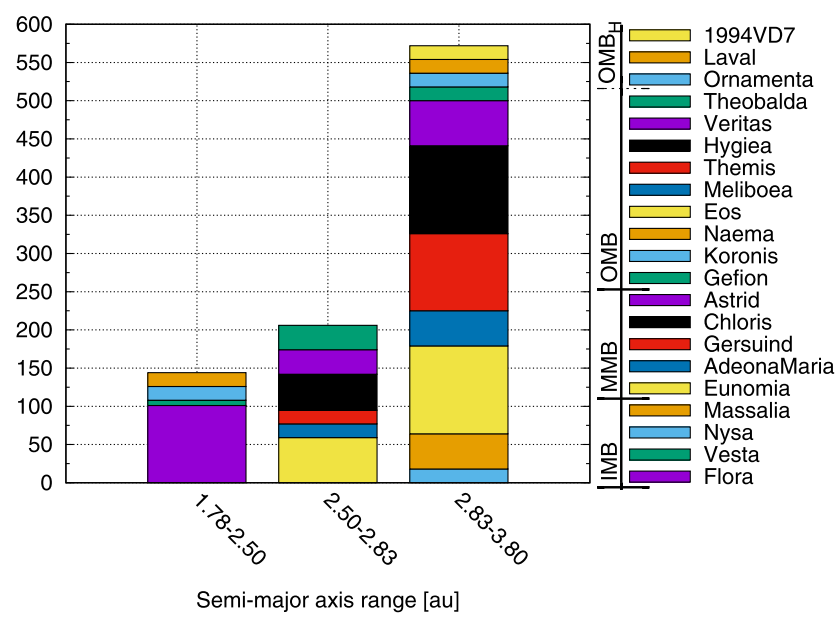

Fig. 6 Number of asteroids that drifted more than $\Delta \geq 3 \times 10^{-4}$ au by family, divided into the 3 regions of the asteroid belt (by semi-major axis): $1.78<a<2.50$ au (IMB, defined before in Table 1 and so on for the other 3 groups): Flora, Vesta, Nysa, Messalia; $2.50 \leq a<2.83$ au (MMB): Eunomia, Adeona-Maria, Gersuing, Chloris, Astrid, Gefion; $2.83 \leq a \leq 3.8$ au (OMB): Koronis and then Naema, Eos, Meliboea, Themis, Hygiea, Veritas, Theobalda, Ornamenta, Laval and $1994 \mathrm{VD}_{7}$ (only this last 3 are highly $(\mathrm{H})$ inclined families: $i>17.16^{\circ}$ )

border of its region), Eos and Themis, in the $O M B$, and Flora in the $I M B$. On a smaller scale (more than 20 but less than 100 deflected asteroids of kilometer sizes, $H<$ 14): Eunomia, Chloris, Astrid and Gefion in the $M M B$, and Eos, Meliboea and Veritas in the $O M B$. Figure 6 clearly shows that the $O M B$ is the most affected region of the main belt.

Although there is still some debate in estimating properly the ages of the asteroid families, much work has been done to establish their ages via dynamical properties of these populations, e.g. Brož et al. (2013), Spoto et al. (2015) and Carruba et al. (2016). These works assert that the eldest families, ones that might have existed in the first 200 Myrs after the end of the LHB are few, i.e. Themis, Maria, Koronis, Ursula, Eunomia and Flora (only the last two families for Carruba et al. 2016). Our results show that all these old families can be perturbed significantly, except for Ursula and Maria, which have a significantly lower number of deflected asteroids with a $\Delta a>K_{\text {kill }}$.

However, we point out that the computation of the age via dynamical properties typically ignores the effects of $\mathrm{C}+\mathrm{TNO}$ close encounters. If $\mathrm{C}+\mathrm{TNO}$ effects are significant at early times, the currently-accepted asteroid family ages of old families can be in part inaccurate, especially for the most affected zones of the Main belt by close encounters with large TNOs in the time span of about $\sim 3-3.8$ Gyrs ago: the main result here is that in the first hundred million years after the $L H B$ main belt families can be significantly perturbed by massive Centaurs and TNOs.

\section{Conclusions}

Centaurs and TNOs do not cause significant deflections with the main belt asteroids at the current time but they can perturb them significantly in the time span from the LHB to the present, especially before 3.5 Gyrs ago. Our results show that only $\mathrm{C}+\mathrm{TNO}$ with a mass larger than $10^{-9} \mathrm{~m}_{\odot}$ can produce an important change of an MBA-orbit, meaning a change $\Delta a \gtrsim K_{\text {kill }}$ au.

Current $\mathrm{C}+\mathrm{TNO}$ s that enter the main belt with a low semi-major axis have a very short average life inside the belt and they escape the soonest. We find that the TNOs of the ancient population reside in the belt longer than the present population, almost twice the time. $23 \%$ of the Centaurs and $3 \%$ of the TNOs enter in the main belt for at least $2 \mathrm{kyrs}$ over the recent 50 Myr time span. Some C+TNOs stay for relatively long periods in the main belt in our simulations, up to 3 Myrs (3.7 Myrs for the AP case), with some loweccentricity orbits, $e \sim 0.1$. Concerning Centaurs, we found that only ones with an initial semi-major axis larger than that of Saturn enter the main belt, and in particular, with $a \gtrsim 13.7$ au.

The orbits of TNOs during their belt-crossing phases resemble those of known large main-belt asteroids, so this could be a suggestion to investigate in more detail whether some dark, primitive main belt asteroids on short-lived orbits may be former TNOs, e.g. some asteroids like (511) Davida show this behavior.

Typical eccentricities and inclinations of $\mathrm{C}+\mathrm{TNO}$ when they reside in the belt are respectively $e \gtrsim 0.16$ and $i \lesssim 25^{\circ}$. Most of the C+TNOs pass initially in the outer main belt (in particular the ancient population) and their semi-major axis is typically $\sim 5 \mathrm{au}$, with the present population having larger values on average, but more present interiorly than the ancient population.

Presently $\mathrm{C}+\mathrm{TNO}$ orbits pass rarely into the inner main belt, they pass mostly through the outer one, generally, with $a=2.6-3.8 \mathrm{au}$. It is important to note that, under our results, billions of years ago, after the Late Heavy Bombardment, massive $\left(m>10^{-9} m_{\odot}\right)$ TNOs would have entered into the inner solar system and with lower eccentricity on average than the present population, having larger probabilities to interact with main belt asteroids.

The young asteroid families currently cannot be perturbed much by even the largest $\mathrm{C}+\mathrm{TNOs}$; the probability that massive bodies arrive now is also low or negligible.

On the contrary, at least $3 \%$ of the main belt can be significantly perturbed in the first hundreds of million of years after the LHB. For this reason some old families and/or former "paleo-families" which may have existed in the past can be modified after the $L H B$ by large minor bodies. Under our model, Flora and Eunomia are the most affected families, therefore their past populations were much larger. If 
we suppose that the estimated age of other old families are biased by neglecting the effects of close encounters with the $\mathrm{C}+\mathrm{TNOs}$, we could assert that those families with underestimated ages might have been perturbed also. Perhaps the Hygiea family, or a paleo-family of Hygiea: from our results a large part of the border of its region in osculating elements was depleted by the passage of large C+TNOs. The most affected region of the belt after the LHB was the Outer main belt and its related families. We lastly underline that Centaurs and TNOs can also disperse part of the main belt families into other dynamical regions, in an interval of time $t \gtrsim 100-200$ Myrs after 3.8 Gyrs ago. They can contribute on diffusing out some asteroids with a typical taxonomic type of its own relative family and mix them with other asteroids of different taxonomic type inside other families, like the case of the C-types in the Hungaria family, and may give rise to small interlopers in families.

Acknowledgements MAG wants to acknowledge the support by the Austrian FWF project P23810-N16 and the "Reitoria de pós-graduação da UNESP" (PROPg, grant PVExt-2015). The core part of this work was done when MAG has been present at UNESP as "visiting fellow". MAG wants also to thanks Prof. V. Carruba for his important suggestions for the paper and Dr. Y. Cavecchi for suggestions in computational improvements and Prof. A. Morbidelli for data on the population decay of the TNOs. SA wants to thank Brazilian National Research Council (CNPq, grant 13/15357-1). This work was also supported in part by the Natural Sciences and Engineering Research Council of Canada.

\section{References}

Adams, E.R., Gulbis, A.A.S., Elliot, J.L., Benecchi, S.D., Buie, M.W., Trilling, D.E., Wasserman, L.H.: Astron. J. 148, 55 (2014). 1311. 3250. doi:10.1088/0004-6256/148/3/55

Bailey, B.L., Malhotra, R.: Icarus 203, 155 (2009). 0906.4795. doi:10. 1016/j.icarus.2009.03.044

Bancelin, D., Hestroffer, D., Thuillot, W.: Celest. Mech. Dyn. Astron. 112, 221 (2012). doi:10.1007/s10569-011-9393-8

Brasser, R., Morbidelli, A.: Icarus 225, 40 (2013). 1303.3098. doi:10. 1016/j.icarus.2013.03.012

Brasser, R., Schwamb, M.E., Lykawka, P.S., Gomes, R.S.: Mon. Not. R. Astron. Soc. 420, 3396 (2012). 1111.7037. doi:10.1111/ j.1365-2966.2011.20264.x

Brown, M.E., Schaller, E.L.: Science 316, 1585 (2007). doi:10.1126/ science. 1139415

Brož, M., Morbidelli, A., Bottke, W.F., Rozehnal, J., Vokrouhlický, D., Nesvorný, D.: Astron. Astrophys. 551, 117 (2013). 1301.6221. doi:10.1051/0004-6361/201219296

Carruba, V., Huaman, M.E., Domingos, R.C., Santos, C.R.D., Souami, D.: Mon. Not. R. Astron. Soc. 439, 3168 (2014). 1401.6332. doi: $10.1093 / \mathrm{mnras} / \mathrm{stu} 192$

Carruba, V., Nesvorný, D., Aljbaae, S., Huaman, M.E: Mon. Not. R. Astron. Soc. 451, 244 (2015). 1505.03745. doi:10.1093/ mnras/stv997

Carruba, V., Nesvorný, D., Aljbaae, S., Domingos, R.C., Huaman, M.: Mon. Not. R. Astron. Soc. 458, 3731 (2016). 1603.00818. doi:10.1093/mnras/stw533

di Sisto, R.P., Brunini, A.: Icarus 190, 224 (2007). doi:10.1016/ j.icarus.2007.02.012 di Sisto, R.P., Brunini, A., de Elía, G.C.: Astron. Astrophys. 519, 112 (2010). 1005.3267. doi:10.1051/0004-6361/200913668

Dones, L., Levison, H.F., Duncan, M.: In: Rettig, T., Hahn, J.M. (eds.) Completing the Inventory of the Solar System. Astronomical Society of the Pacific Conference Series, vol. 107, p. 233 (1996)

Eggl, S., Dvorak, R.: In: Souchay, J., Dvorak, R. (eds.) Dynamics of Small Solar System Bodies and Exoplanets. Lecture Notes in Physics, vol. 790, p. 431. Springer, Berlin (2010). doi:10.1007/ 978-3-642-04458-8_9

Fernández, J.A., Sosa, A.: Planet. Space Sci. 118, 14 (2015). doi:10. 1016/j.pss.2015.07.010

Fernández, J.A., Gallardo, T., Brunini, A.: Icarus 172, 372 (2004). doi:10.1016/j.icarus.2004.07.023

Fuentes, C., Trilling, D.E., Schlichting, H.: In: AAS/Division for Planetary Sciences Meeting Abstracts. AAS/Division for Planetary Sciences Meeting Abstracts, vol. 46, p. 421 (2014)

Galiazzo, M.A.: The threat of NEA's and the origin of terrestrial planets impactors. PhD thesis, University of Vienna (2013)

Galiazzo, M.A., Schwarz, R.: Mon. Not. R. Astron. Soc. 445, 3999 (2014). doi:10.1093/mnras/stu2016

Galiazzo, M.A., Bazsó, Á., Dvorak, R.: Planet. Space Sci. 84, 5 (2013). doi:10.1016/j.pss.2013.03.017

Galiazzo, M.A., Bazsó, Á., Huber, M.S., Losiak, A., Dvorak, R., Koeberl, C.: Astron. Nachr. 334, 936 (2013). 1305.3631. doi:10.1002/ asna.201211964

Galiazzo, M.A., Bazso, A., Dvorak, R.: Mem. Soc. Astron. Ital. Suppl. 26, 38 (2014)

Gladman, B., Kavelaars, J.J., Petit, J.-M., Morbidelli, A., Holman, M.J., Loredo, T.: Astron. J. 122, 1051 (2001). doi: $10.1086 / 322080$

Gladman, B., Marsden, B.G., Vanlaerhoven, C.: In: Barucci, M.A., Boehnhardt, H., Cruikshank, D.P., Morbidelli, A., Dotson, R. (eds.) Nomenclature in the Outer Solar System, p. 43 (2008)

Hanslmeier, A., Dvorak, R.: Astron. Astrophys. 132, 203 (1984)

Hartmann, W.K., Ryder, G., Dones, L., Grinspoon, D.: In: Canup, R.M., Righter, K., et al.(eds.) The Time-Dependent Intense Bombardment of the Primordial Earth/Moon System, p. 493 (2000)

Horner, J., Evans, N.W., Bailey, M.E.: Mon. Not. R. Astron. Soc. 354, 798 (2004a). astro-ph/0407400. doi:10.1111/ j.1365-2966.2004.08240.x

Horner, J., Evans, N.W., Bailey, M.E.: Mon. Not. R. Astron. Soc. 355, 321 (2004b). astro-ph/0408576. doi:10.1111/j.13652966.2004.08342.x

Huaman, M.E., Carruba, V., Domingos, R.C.: Mon. Not. R. Astron. Soc. 444, 2985 (2014). 1408.7080. doi:10.1093/mnras/stu 1655

Kirchoff, M.R., Chapman, C.R., Marchi, S., Curtis, K.M., Enke, B., Bottke, W.F.: Icarus 225, 325 (2013). doi:10.1016/ j.icarus.2013.03.018

Knežević, Z., Milani, A.: Astron. Astrophys. 403, 1165 (2003). doi:10. 1051/0004-6361:20030475

Levison, H.F., Duncan, M.J.: Astrophys. J. Lett. 406, 35 (1993). doi:10. $1086 / 186780$

Levison, H.F., Duncan, M.J.: Icarus 127, 13 (1997). doi:10.1006/ icar.1996.5637

Lykawka, P.S., Horner, J., Jones, B.W., Mukai, T.: Mon. Not. R. Astron. Soc. 398, 1715 (2009). 0909.0404. doi:10.1111/ j.1365-2966.2009.15243.x

Migliorini, F., Zappalà, V., Vio, R., Cellino, A.: Icarus 118, 271 (1995). doi:10.1006/icar.1995.1191

Morbidelli, A.: Icarus 127, 1 (1997). doi:10.1006/icar.1997.5681

Napier, W.M.: Mon. Not. R. Astron. Soc. 448, 27 (2015). 1503.04451. doi:10.1093/mnras/stu2681

Nesvorny, D.: NASA Planetary Data System 189 (2012)

Nesvorný, D., Bottke, W.F. Jr., Dones, L., Levison, H.F.: Nature 417, 720 (2002)

Novaković, B., Cellino, A., Knežević, Z.: Icarus 216, 69 (2011). 1108.3740. doi:10.1016/j.icarus.2011.08.016 
Rabinowitz, D.L., Schaefer, B.E., Tourtellotte, S.W.: Astron. J. 133, 26 (2007). astro-ph/0605745. doi:10.1086/508931

Schwamb, M.E., Brown, M.E., Fraser, W.C.: Astron. J. 147, 2 (2014). 1310.7049. doi:10.1088/0004-6256/147/1/2

Spoto, F., Milani, A., Knežević, Z.: Icarus 257, 275 (2015). 1504. 05461. doi:10.1016/j.icarus.2015.04.041

Tedesco, E.F., Veeder, G.J., Fowler, J.W., Chillemi, J.R.: The IRAS minor planet survey. Technical report (Dec. 1992)
Tiscareno, M.S., Malhotra, R.: Astron. J. 126, 3122 (2003). astro-ph/0211076. doi:10.1086/379554

Volk, K., Malhotra, R.: Icarus 224, 66 (2013). 1211.2774. doi:10. 1016/j.icarus.2013.02.016

Warner, B.D., Harris, A.W., Vokrouhlický, D., Nesvorný, D., Bottke, W.F.: Icarus 204, 172 (2009). doi:10.1016/j.icarus.2009.06.004

Zappalà, V., Bendjoya, P., Cellino, A., Farinella, P., Froeschlé, C.: Icarus 116, 291 (1995). doi:10.1006/icar.1995.1127 